\title{
Ultrasound-guided interventions for painful shoulder: from anatomy to evidence
}

This article was published in the following Dove Press journal: Journal of Pain Research

\author{
Ke-Vin Chang ${ }^{1,2}$ \\ Kamal Mezian ${ }^{3}$ \\ Ondřej Naňka ${ }^{4}$ \\ Wei-Ting $\mathrm{Wu}^{\prime}$ \\ Chih-Peng $\operatorname{Lin}^{5,6}$ \\ Levent Özçakar ${ }^{7}$
}

'Department of Physical Medicine and Rehabilitation, National Taiwan University Hospital, Bei-Hu Branch, Taipei, Taiwan; ${ }^{2}$ Department of Physical Medicine and Rehabilitation, National Taiwan University, College of Medicine, Taipei, Taiwan; ${ }^{3}$ Department of Rehabilitation Medicine, Charles University, First Faculty of Medicine, Prague, Czech Republic; ${ }^{4}$ Institute of Anatomy, Charles University, First Faculty of Medicine, Prague, Czech Republic; ${ }^{5}$ Department of Anesthesiology, National Taiwan University Hospital, Taipei, Taiwan; ${ }^{6}$ Department of Anesthesiology, National Taiwan University, College of Medicine, Taipei, Taiwan; ${ }^{7}$ Department of Physical and Rehabilitation Medicine, Hacettepe University Medical School, Ankara, Turkey

Correspondence: Ke-Vin Chang Department of Physical Medicine and Rehabilitation, National Taiwan University Hospital, Bei-Hu Branch and National Taiwan University College of Medicine, Taipei, Taiwan Email kvchang0I I@gmail.com

Chih-Peng Lin

Department of Anesthesiology, National Taiwan University Hospital, National

Taiwan University College of Medicine,

Taipei, Taiwan

Email cplin0I23@gmail.com

\begin{abstract}
Shoulder pain is a common musculoskeletal disorder of variable etiology, ranging from rotator cuff pathology to peripheral nerve entrapment. Advances in ultrasound (US) technology have allowed static and dynamic evaluation of shoulder problems and most importantly, offer real-time, radiation-free guidance for interventions. The present review aims to describe shoulder anatomy in detail using information from cadaveric models and to illustrate US-guided techniques using clearly labeled figures and videos. The review will also present evidence of specific US-guided therapies for shoulder pain by summarizing landmark studies, systematic reviews, and meta-analyses. The following shoulder structures will be covered: 1) the biceps long head tendon, 2) the acromioclavicular joint, 3) the subacromial-subdeltoid bursa, 4) the glenohumeral joint, 5) the suprascapular nerve, and 6) the axillary nerve.
\end{abstract}

Keywords: pain, sonography, tendon, joint, injection, rehabilitation

\section{Introduction}

Shoulder pain is a common musculoskeletal disorder, with a lifetime prevalence of $6.7 \%-66.7 \%$ in the general population. ${ }^{1}$ The causes of chronic shoulder pain are variable, ranging from rotator cuff pathology to peripheral nerve entrapment. ${ }^{2,3}$ Advances in ultrasound (US) technology allow static and dynamic evaluation of shoulder problems and offer real-time, radiation-free guidance for therapeutic interventions. ${ }^{4}$ The main philosophy behind these interventions starts with "seeing is believing" and continues with "once seen, can be accessed." Undoubtedly, US guidance provides an additional margin of safety for interventional techniques. Depending on target localization, physicians can easily select the technique of needle insertion and thereby avoid injury to nearby structures. It is important to consider that US guidance for interventions should not be considered as precise targeting alone; it offers comprehensive guidance for the treatment process starting with clinical decision making. ${ }^{5}$

A successful intervention for complicated shoulder pathology should be based on familiarization with the three-dimensional regional anatomy, US-guided injection techniques, and evidence of therapeutic effectiveness. The present review aims to describe shoulder anatomy in detail using information from cadaveric models and illustrates US-guided techniques using clearly labeled figures and videos. The evidence for specific US-guided therapies for shoulder pain is also presented by summarizing landmark studies, systematic reviews, and meta-analyses. We aim to focus on the following target structures: 1) the long head of the biceps tendon, 2) the acromioclavicular (AC) joint, 3) the subacromial-subdeltoid bursa, 4) the glenohumeral joint, 5) the suprascapular nerve, and 6) the axillary nerve. 


\section{Long head of the biceps tendon Anatomy}

The biceps brachii muscle has two proximal tendons. The long head tendon originates from the supraglenoid tubercle of the scapula, while the short head tendon is attached to the coracoid process (Figure 1A). Because the proximal long head tendon courses through the glenohumeral joint, it has an intimate association with the rotator cuff tendon pathology, and it is more likely to cause pain than the short head tendon. After the long head tendon leaves the superior labrum, it runs in the rotator cuff interval, a space bordered by the subscapularis tendon anteriorly, supraspinatus tendon posteriorly, coracohumeral ligament superiorly, and superior glenohumeral ligament inferiorly. ${ }^{6}$ Once the tendon exits the rotator cuff interval, it passes into the bicipital groove on the humeral head, between the lesser tuberosity medially and the greater tuberosity laterally. At the groove level, the tendon is stabilized by the transverse humeral ligament, an extension of the fibers of the subscapularis tendon. The long head of the biceps tendon is enveloped by the biceps tendon sheath, which is an extension of the synovial sheath of the glenohumeral joint. The tendon is supplied by the ascending branch of the anterior circumflex humeral artery. ${ }^{?}$

\section{US imaging and guided injection technique \\ At the level of the intertubercular groove}

The patient is seated with the arm in neutral position by the side. External rotation of the arm allows the intertubercular groove to face more anterolaterally. The transducer is placed horizontally, lateral to the coracoid process. The long head tendon can be visualized in the bicipital groove between the greater and lesser tuberosities of the humeral head. ${ }^{8} \mathrm{It}$ is seen underneath the transverse humeral ligament, which appears as a hyperechoic linear structure bridging the two aforementioned tuberosities. On the power Doppler mode, the ascending branch of the anterior circumflex humeral artery can be enhanced at the lateral aspect of the bicipital groove and can easily be avoided during injection. ${ }^{7}$
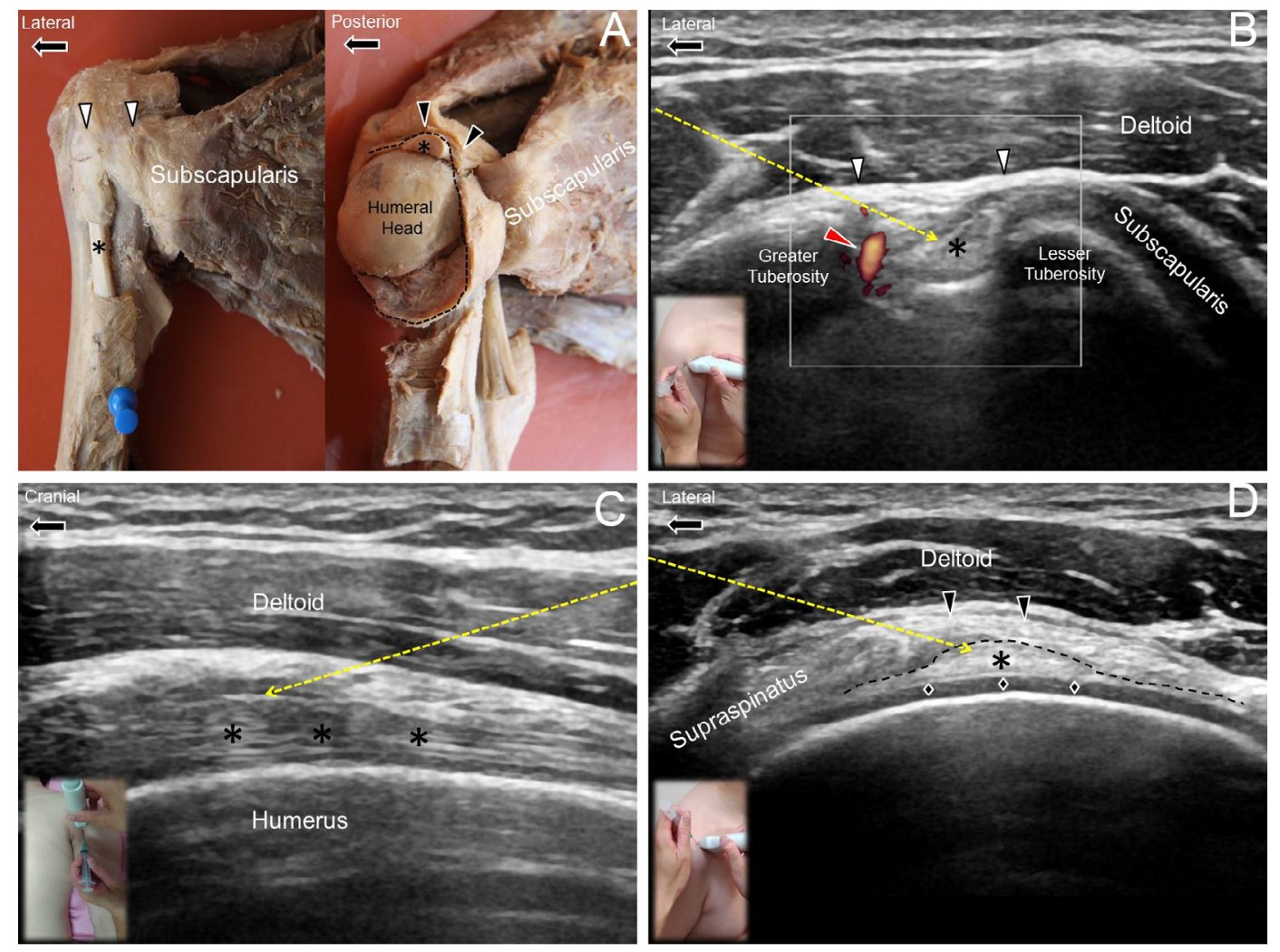

Figure I Ultrasound guided injection for the long head of the biceps tendon

Notes: (A) Cadaver models of the biceps long head tendon. The biceps tendon (asterisk) is covered by the transverse humeral ligament (white arrowheads) in the intertubercular groove level (left image). The biceps tendon is below the coracohumeral ligament (black arrowheads) and the glenohumeral capsule (dashed line) in the rotator cuff interval level (right image). (B) US-guided in-plane approach to target the tendon in short axis at the intertubercular groove level. Red arrowhead shows ascending branch of the anterior humeral circumflex artery. White arrowheads indicate the transverse humeral ligament. (C) US-guided in-plane approach to target the tendon in long axis at the intertubercular groove level. (D) US-guided in-plane approach to target the tendon in short axis at the rotator cuff interval level. Diamonds show the hyaline cartilage. Yellow dashed arrow: the needle trajectory. Asterisks indicate the long head of the biceps tendon. Black arrowheads indicate the coracohumeral ligament. The dashed line indicates the glenohumeral capsule. 
During injection to the short-axis of the tendon, it is recommended to position the target in the lateral one-third of the screen. This can effectively shorten the needle trajectory through the deltoid muscle. The needle is then introduced from lateral to medial by an in-plane approach at an oblique angle of 30-45 degrees. After piercing the transverse humeral ligament, the needle reaches the biceps tendon sheath. By slow injection, a gradual distention can be observed, with the tendon surrounded by the injectate, described as the "doughnut sign," indicating a successful intrasheath injection (Figure 1B, Video S1).

Alternatively, the tendon can be injected along its long axis. First, the patient should be either supine or in the sitting position. The transducer is placed in the sagittal plane in line with the long axis of the tendon. The needle is then advanced by an in-plane approach from caudal to cranial (Figure 1C, Video S1). It is recommended to place the needle tip just above the superficial portion of the tendon. This is to avoid misplacement of the needle inside the subdeltoid bursa that is located underneath the deltoid muscle and overlies the transverse humeral ligament.

\section{Rotator cuff interval level}

The patient is seated with the arm internally rotated and extended with the volar side of the hand placed on the buttock. The transducer is first positioned on the bicipital groove and moved cranially until the long head tendon is interposed between the subscapularis tendon anteriorly and the supraspinatus tendon posteriorly. The needle is then introduced from lateral to medial by an in-plane approach, targeting the tendon underneath the coracohumeral ligament (Figure 1D, Video S1). ${ }^{9}$ Compared with biceps tendon injection at the intertubercular groove level, more injectate may flow back to the glenohumeral joint, rendering this technique suitable for patients with concomitant adhesive capsulitis.

\section{Evidence}

Petscavage-Thomas and Gustas conducted a retrospective study to compare the effectiveness of US- vs fluoroscopyguided biceps tendon injection at the groove level. ${ }^{10}$ In this study, US guidance had a higher success rate with administration of medication inside the tendon sheath. There was no significant difference in pain relief and complication rates. Stone and Adler reported a 100\% technical success rate with injection into the long head tendon at the rotator cuff interval confirmed by intrasheath fluid on postinjection images. ${ }^{9}$ Although both these studies established the precision of US-guided injection into the long head of the biceps tendon, the main concern is the timing of this intervention. Chang et al found that bicipital peritendinous effusion and biceps tendon morphology were related to various types of rotator cuff pathology and might also be a consequence of subacromial impingement. ${ }^{11-13}$ Furthermore, another study pointed out that shoulder pathology manifesting as pain on the bicipital groove was more likely to show higher power Doppler signals near the lesser tuberosity. ${ }^{7}$ Therefore, the decision to perform biceps tendon injection should be based on both clinical findings and ultrasonographic imaging.

\section{AC joint Anatomy}

The $\mathrm{AC}$ joint is a diarthrodial joint that connects the clavicle and the acromion. It is reinforced by a capsule with synovial lining and has articular cartilage at the bone ends (Figure 2A, B). The joint capsule is formed by four AC ligaments - anterior, posterior, superior, and inferior; the superior ligament is considered to be thicker than the others. ${ }^{14,15}$ There is usually an articular disc interposed between the clavicular and acromial ends, which undergoes significant degeneration after the age of 40 years. ${ }^{16}$ The $\mathrm{AC}$ joint plays a substantial role in the maintenance of horizontal stability during shoulder movements.

\section{US imaging and guided injection technique}

The patient is seated with the arm hanging by the side. The lateral edge of the clavicle can easily be palpated; the gap adjacent to the lateral end of the clavicle is the AC joint space. The transducer is placed in the coronal plane, bridging the joint. The superior $\mathrm{AC}$ ligament can be seen as a hyperechoic fibrillary arc demarcating the superior joint capsule, and a meniscus-like disc is visualized between the acromial and clavicular ends.

There are three common techniques used for US-guided $\mathrm{AC}$ joint injection. The out-of-plane approach is perhaps the most commonly used method. Initially, the AC joint is visualized at the center of the image. The needle is introduced from anterior to posterior until its tip is seen as a hyperechoic spot below the superior AC ligament (Figure 2C, Video S2). The anterior-to-posterior approach is preferred over the posterior-to-anterior approach because the anterior joint gap is wider than the posterior gap. ${ }^{16}$ The needle should not be inserted too deep as it may pierce the intra-articular meniscus and the inferior $\mathrm{AC}$ capsule, with flow of injectate into the subacromial bursa.

The second technique is using an in-plane approach. The transducer is placed along the coronal plane as described 

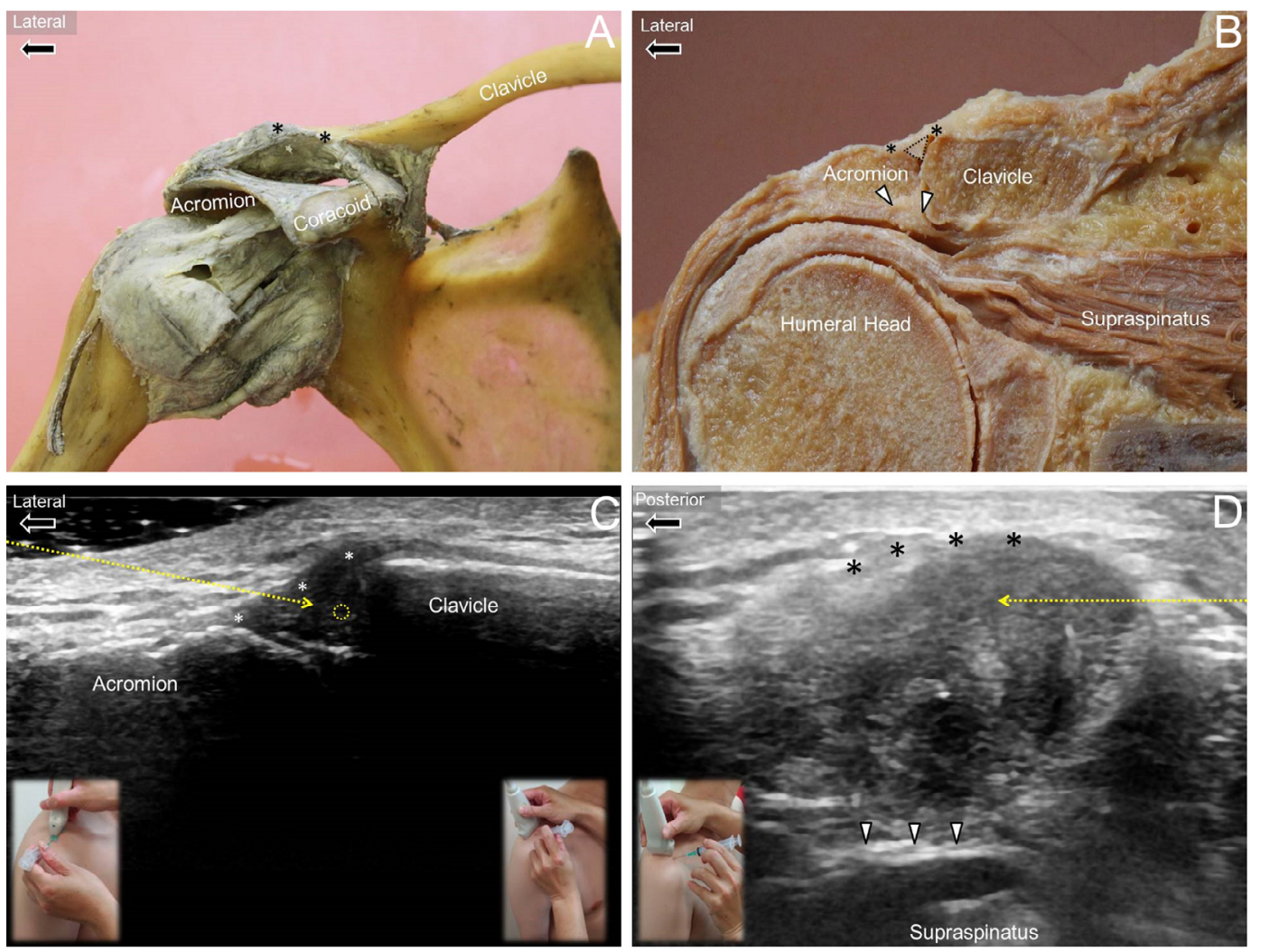

Figure 2 Ultrasound guided injection for the acromioclavicular joint

Notes: (A) Cadaver model of the acromioclavicular joint, stabilized by ligaments connecting the acromion, the coracoid process, and the clavicle. (B) Coronal cross-section of a cadaveric acromioclavicular joint. Dashed triangle indicates the articular disc and white arrowheads point to the inferior acromioclavicular ligament. (C) US-guided inplane (dashed arrow) and out-of-plane (dashed circle) approaches to the coronal section of the acromioclavicular joint. (D) US-guided in-plane (dashed arrow) approach to the sagittal section of the acromioclavicular joint. Black (white in Figure 2C). Asterisks: the superior acromioclavicular ligament. The asterisks indicate the superior acromioclavicular ligament. The white arrowheads in $\mathbf{B}$ and $\mathbf{D}$ indicate the inferior acromioclavicular ligament.

above. The lateral edge of the transducer is raised from the skin surface, and the gap so created is filled with sterile coupling gel (Figure 2C, Video S2). The needle is introduced from lateral to medial through the gel until the joint capsule is pierced.

The third technique is also an in-plane approach. Unlike the second, the transducer is placed along the sagittal plane of the AC joint. The articular disc appears circular on this view. The needle is introduced from anterior to posterior until it passes through the superior AC ligament (Figure 2D, Video S2).

\section{Evidence}

Although the AC joint is superficial, precise injection into the joint space may be difficult to ascertain. Peck et al conducted a prospective study comparing US- vs palpation-guided injection of AC joints on cadavers and found that the success rate of intra-articular administration was $100 \%$ in the US-guided group compared with only $40 \%$ in the palpationguided group. ${ }^{17} \mathrm{~A}$ similar cadaveric study was performed by Borbas et al in which the success rate of intra-articular injection was $90 \%$ in the US-guided group compared with $70 \%$ in the blind injection group. ${ }^{18}$ Park et al, in a subsequent retrospective analysis, showed greater improvement in pain and shoulder function using an US-guided approach compared with the palpation technique. ${ }^{19}$ On the contrary, two randomized controlled trials reported that although US guidance led to a higher success rate of intra-articular injection, the clinical outcome was not better than periarticular or palpation-guided injections. ${ }^{20,21}$ The above studies suggest that while US-guided AC joint injection definitely allows precise intra-articular administration of the injectate, there is still uncertainty regarding treatment outcomes compared with the palpation-guided approach.

\section{Subacromial-Subdeltoid Bursa Anatomy}

The subacromial-subdeltoid bursa is located below the acromion, the coracoacromial ligament, and the deltoid muscle and is superficial to the supraspinatus tendon. It is a large 
synovium-lined structure expanding from the subcoracoid space medially to the subdeltoid region overlying the bicipital groove laterally (Figure 3A). The distal boundary of the bursa is located between the greater tuberosity of the humerus and the deltoid tuberosity. It usually has a direct connection with the subcoracoid bursa but not with the subscapularis recess that is an extension of the glenohumeral joint. ${ }^{22}$

\section{US imaging and guided injection technique}

There are two methods of US-guided subacromial-subdeltoid bursa injection, both using an in-plane technique. In the first method, the transducer is initially placed in the coronal plane along the long axis of the supraspinatus tendon. The subacromial-subdeltoid bursa can be visualized as an anechoic or hypoechoic linear structure, originating from the subacromial region and extending to the area distal to the insertion of the supraspinatus tendon. The outer and inner layers of the bursa are usually outlined by peribursal fat that appears as two hyperechoic strips. The needle is introduced from caudal to cranial until the tip reaches the space interposed between the deltoid muscle and the supraspinatus tendon (Figure 3B, Video S3). ${ }^{23}$

In the second technique, the transducer is placed along the short axis of the supraspinatus tendon. In this view, the supraspinatus tendon looks like a rainbow arc overlying the humeral cortex. The needle can be introduced from lateral to medial to target the subacromial-subdeltoid bursa between the deltoid muscle and the supraspinatus tendon (Figure 3C, Video S3) ${ }^{24}$

During both interventions, the injectate should appear to be evenly distributed within the bursa. If there is a localized accumulation of the injectate, it may indicate multiple compartments caused by bursal adhesion. If multiple compartments are present, the needle needs to be moved back and forth to break down the adhesions either before or during the administration of the medication.

\section{Evidence}

In 2015, Wu et al conducted a systematic review and meta-analysis comparing the effectiveness of US- vs landmark-guided corticosteroid injection within the subacromial-subdeltoid bursa. ${ }^{25}$ They included seven trials with 445 patients and found that the former technique resulted in greater functional improvement of the shoulder. However, a subsequent randomized controlled trial of patients with subacromial impingement syndrome reported no difference in pain reduction and functional improvement with or without US guidance. ${ }^{26}$ The contradictory results may be due to different patient populations and symptom severity. The thickness of the subdeltoid bursa, dynamic subacromial impingement assessment, the side involved, and response to subacromial lidocaine injection have all been reported as predictors of success with US-guided subacromial-subdeltoid bursa injection and thus should routinely be assessed before the interventions. ${ }^{27}$

\section{Glenohumeral joint Anatomy}

The glenohumeral joint is a ball-and-socket type diarthrodial structure, lined by synovium and reinforced by the glenohumeral capsule. The latter is made of thick connective tissue, originating from the bony glenoid and labrum, and inserts on the anatomic neck of the humerus. There are several redundant and lax portions of the glenohumeral capsule, including the anterior, posterior, and axillary recesses, which allow multiplanar motion of the glenohumeral joint (Figure 4A, B, C). ${ }^{28}$

\section{US imaging and guided injection technique}

\section{Anterior glenohumeral joint}

The patient lies supine, and the transducer is placed in the horizontal plane at the level of the coracoid process. Because the anterior glenohumeral joint is located deep, a curvilinear transducer may be required. The anterior glenohumeral joint is seen between the humeral head and the anterior glenoid. There are two US-guided approaches for injection of the anterior glenohumeral joint. The first technique uses an out-of-plane approach. The needle is introduced from caudal to cranial until the gap between the humeral head and the glenoid is reached (Figure 4D, Video S4). The cranial-to-caudal direction is not recommended because the brachial plexus may be injured in the axillary region. The second technique uses an in-plane approach with the needle introduced from lateral to medial, and the tip is advanced to the glenohumeral capsule (Figure 4D, Video S4). This approach is far more difficult because the needle trajectory is easily blocked by the convex humeral head. In addition, needle visibility is usually suboptimal because of the sharp insertion angle. The authors strongly suggest that physicians initially acquire sufficient expertise with the first method in their practice before attempting the second technique.

\section{Superior glenohumeral joint}

Injection to the superior glenohumeral joint is generally indicated in patients with a superior-labrum-anterior-andposterior lesion in order to promote regeneration using 

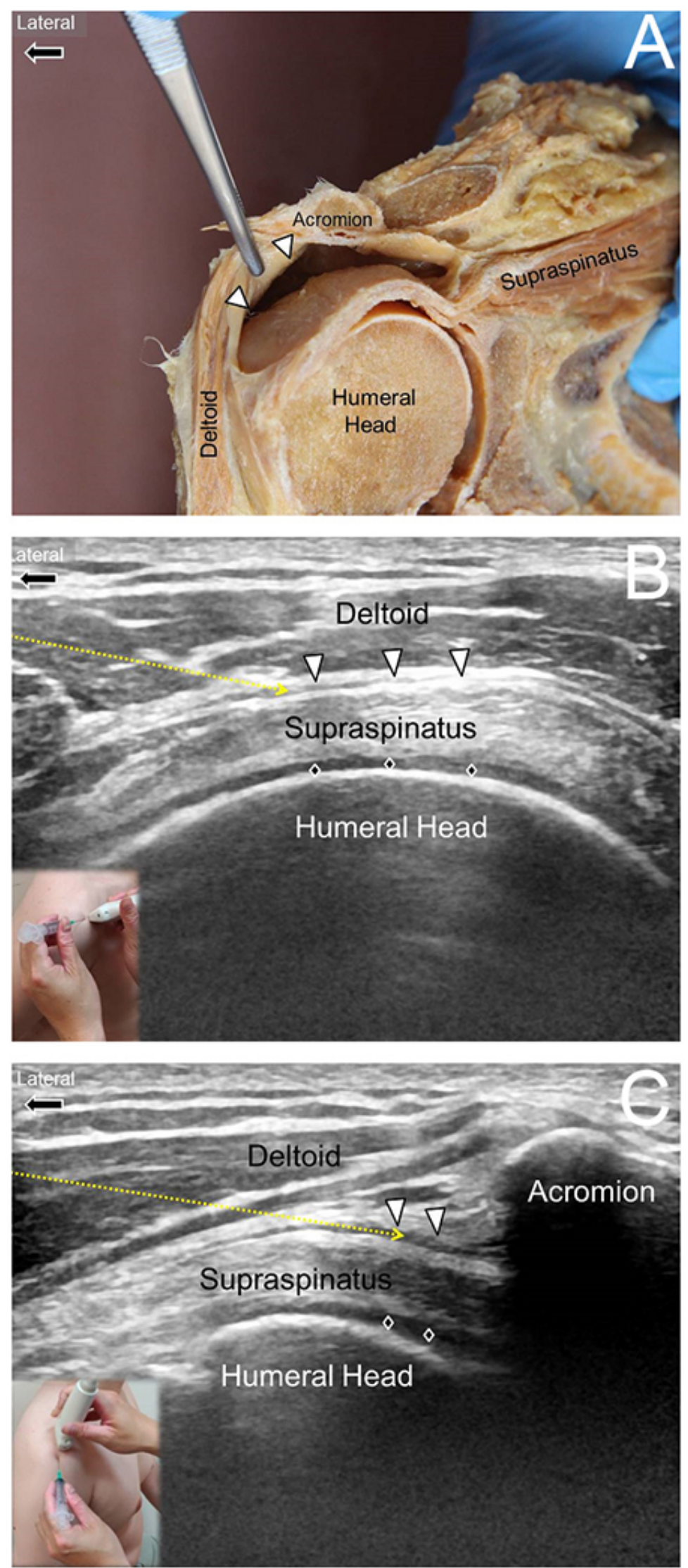

Figure 3 Ultrasound guided injection for the subdeltoid-subacromial bursa

Notes: (A) Cadaver model of the subdeltoid-subacromial bursa (white arrowheads). (B) US-guided in-plane (dashed arrow) approach to the subdeltoid-subacromial bursa (white arrowheads) along the short axis of the supraspinatus tendon. (C) US-guided in-plane (dashed arrow) approach to the subdeltoid-subacromial bursa (white arrowheads) along the long axis of the supraspinatus tendon. Diamonds: hyaline cartilage. 

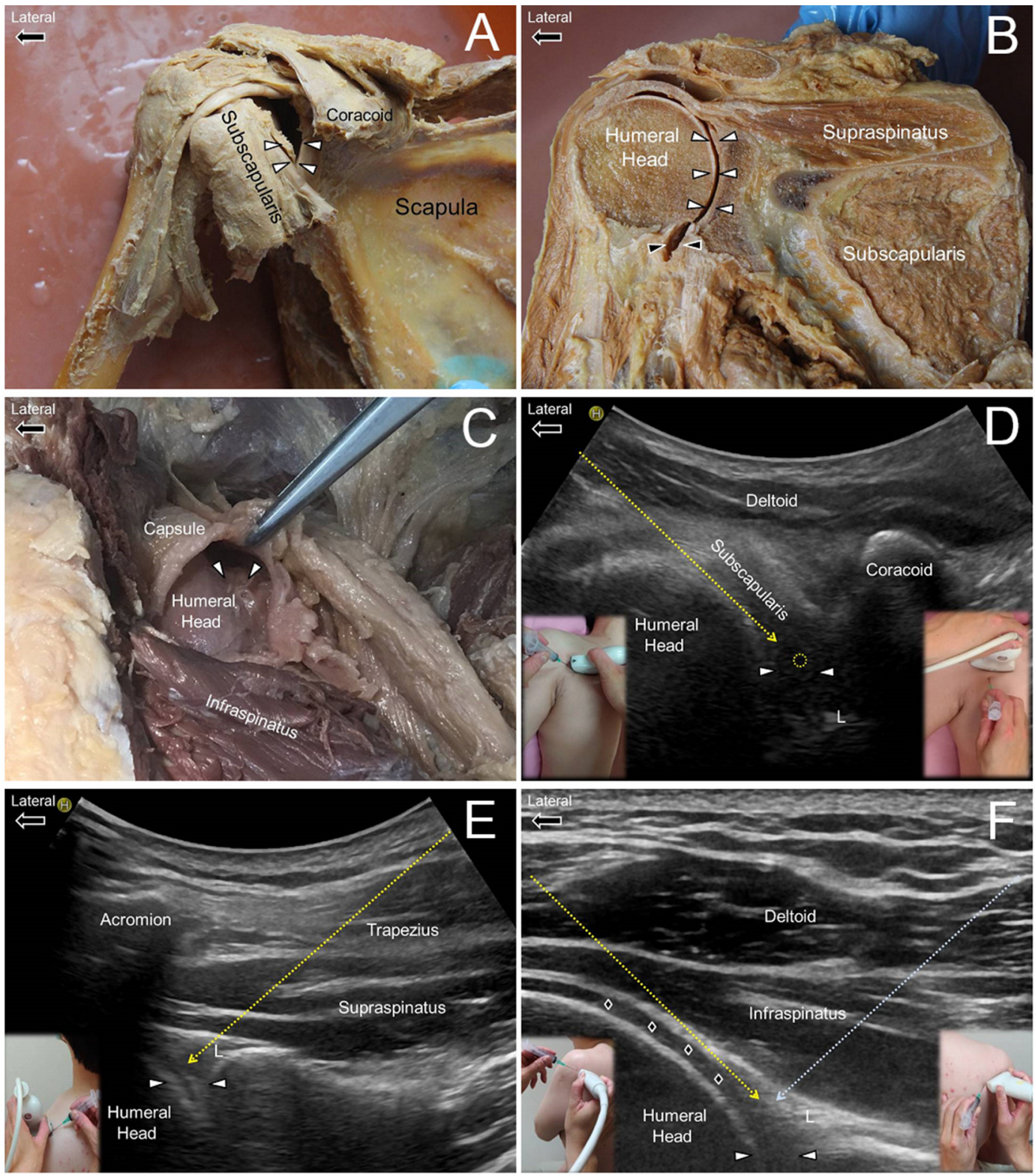

Figure 4 Ultrasound guided injection for the glenohumeral joint

Notes: (A) Anterior aspect of a cadaver model of the glenohumeral joint (white arrowheads). (B) Coronal cross-section of the glenohumeral joint (white arrowheads) and its extension, the axillary pouch (black arrowheads). (C) Posterior aspect of the glenohumeral joint (white arrowheads) and its overlying capsule. (D) US-guided in-plane (dashed arrow) and out-of-plane (dashed circle) approaches to the anterior glenohumeral joint (white arrowheads). (E) US-guided in-plane approach (dashed arrow) to the superior glenohumeral joint (white arrowheads). (F) US-guided in-plane approach from lateral to medial (yellow dashed line) or medial to lateral (blue dashed line) for the posterior glenohumeral joint (white arrowheads). Diamonds: hyaline cartilage.

platelet-rich plasma and dextrose. ${ }^{29}$ During the injection, the patient is seated and the transducer is placed in the coronal plane on the supraspinatus fossa. Because the superior glenohumeral joint is below the acromion, a curvilinear transducer is required; the side beam helps improve visibility of the superior labrum. The needle is introduced by an in-plane approach from medial to lateral until the needle tip passes the lateral edge of the superior labrum (Figure 4E, Video S4).

\section{Posterior glenohumeral joint}

The patient is seated, and the transducer is placed caudal and parallel to the scapular spine. The joint space is visualized between the humeral head and the posterior bony glenoid. There are two commonly applied, in-plane injection techniques for the posterior glenohumeral joint. With the first method, the needle is introduced from medial to lateral until the needle tip pierces the joint capsule lateral to the posterior labrum (Figure 4F, Video S4). In the second method, the 
needle is introduced through the infraspinatus muscle from lateral to medial until the needle tip reaches the junction between the humeral head cartilage and the lateral edge of the posterior labrum (Figure 4F, Video S4). At this point, although the needle shaft is visualized more easily due to a less steep angle of injection, the needle trajectory can be hindered by the convex humeral head if the lateral-to-medial direction is used.

The most common indication for glenohumeral joint injection is adhesive capsulitis where the intracapsular pressure is usually increased. ${ }^{30}$ Therefore, the injection should be carried out slowly while the needle is gradually advanced to prevent the tip from being ejected backward due to increased intra-articular pressure; if not, the intracapsular injection may be ineffective as the injectate may be inadvertently administered into the infraspinatus muscle.

\section{Evidence}

In 2012, Patel et al, in a cadaver study, compared the accuracy of the US-guided posterior approach with the glenohumeral joint with the blind technique on 80 shoulder specimens. The accuracy rate was significantly higher with US guidance compared with blind administration $(92.5 \%$ vs $72.5 \%$, $P=0.02) .{ }^{31}$ In 2014, Amber et al conducted a meta-analysis comparing the precision of US vs fluoroscopy guidance for glenohumeral injections. ${ }^{32}$ They found that US guidance was more accurate although the difference was nonsignificant. Regarding the comparison of anterior vs posterior US-guided approach, Kim et al reported shorter positioning time using the anterior approach but greater pain reduction using the posterior approach. ${ }^{33}$ For treating adhesive capsulitis, a recent meta-analysis indicated that the injection of a huge amount of fluid into the glenohumeral joint (hydrodilatation) may be beneficial in the early recovery of limitation of range of motion, especially in external rotation. ${ }^{34}$ Furthermore, corticosteroids can be added in the regimen used for hydrodilatation to prevent the inflammatory reaction on the glenohumeral capsule.

\section{Suprascapular nerve Anatomy}

The suprascapular nerve arises from the upper trunk of the brachial plexus and receives fibers from $\mathrm{C} 5$ and $\mathrm{C} 6$ nerve roots (Figure 5A). The nerve courses beside the inferior belly of the omohyoid muscle and enters the supraspinatus fossa through the suprascapular notch below the superior transverse suprascapular ligament (Figure 5B). The nerve is accompanied by the suprascapular artery, which usually runs above the transverse suprascapular ligament. The nerve leaves the supraspinatus fossa and reaches the infraspinatus fossa through the spinoglenoid notch (Figure 5C). It provides sensory supply to the AC and glenohumeral joints and also innervates the supraspinatus and infraspinatus muscles. ${ }^{35}$

\section{US imaging and guided injection technique}

\section{Brachial plexus level}

This technique targets the proximal suprascapular nerve and is suitable for patients who are not able to sit steady or for those who suffer entrapment due to the overlying tight trapezius muscle. The patient is supine with the head turned to the opposite side, and the transducer is placed along the sagittal plane on the supraclavicular fossa. The brachial plexus can be seen posterior to the subclavian artery, and the upper trunk is located at the most superior and posterior part of the nerve bundle. Repositioning the transducer more laterally allows visualization of the suprascapular nerve leaving the upper trunk. ${ }^{36}$ The needle can be introduced by an in-plane approach from lateral to medial until the perineurium is reached (Figure 5D, Video S5).

\section{Supraspinatus fossa level}

The patient is seated, and the transducer is placed on the supraspinatus fossa along the scapular plane. The midpoint of the transducer is adjusted to align with the lateral one-third of the scapular spine. The suprascapular nerve is located at the bottom of the supraspinatus fossa. The suprascapular artery can be visualized as a pulsating dot with positive Doppler signals beside the nerve. The needle is introduced from medial to lateral by an in-plane approach until the needle tip pierces the deep fascia of the supraspinatus muscle or the transverse suprascapular ligament (Figure 5E, Video S5). ${ }^{23}$ The injectate is instilled into the floor of the supraspinatus fossa.

\section{Infraspinatus fossa level}

The patient is seated with the arm by the side. The transducer is placed slightly caudal to the scapular spine in the horizontal plane. Unlike visualization of the posterior glenohumeral joint, the medial edge of the transducer needs to be pivoted more cranially to visualize the spinoglenoid notch. The power Doppler mode may be used to locate the suprascapular artery prior to injection. The needle is introduced from medial to lateral until the needle tip reaches the spinoglenoid notch (Figure 5F, Video S5). 

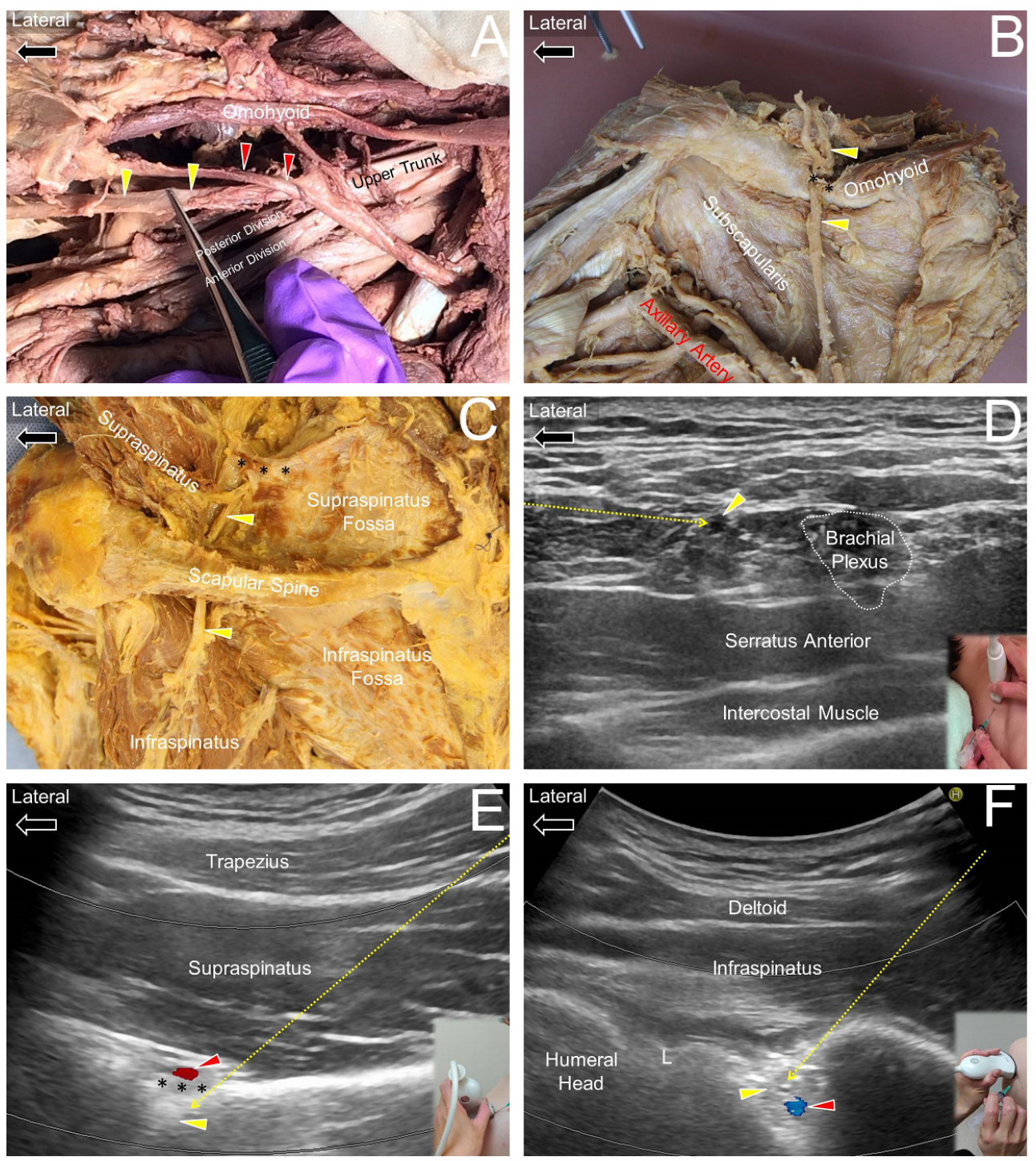

Figure 5 Ultrasound guided injection for the suprascapular nerve

Notes: (A) Cadaver model of the suprascapular nerve (yellow arrowheads) branching from the upper trunk of the brachial plexus. Red arrowheads show the suprascapular artery. (B) The suprascapular nerve (yellow arrowheads) at the entrance the suprascapular fossa. Black asterisks show the superior transverse scapular ligament. (C) The suprascapular nerve (yellow arrowhead) coursing through the supraspinatus fossa and reaching the infraspinatus fossa. Black asterisks indicate the transverse scapular ligament. (D) US-guided in-plane (dashed arrow) approach to the suprascapular nerve (yellow arrowhead) at the brachial plexus level. (E) US-guided in-plane (dashed arrow) approach to the suprascapular nerve (yellow arrowhead) at the supraspinatus fossa level. Black asterisks indicate the transverse scapular ligament. Red arrowheads indicates the suprascapular artery. (F) US-guided in-plane (dashed arrow) approach to the suprascapular nerve at the infraspinatus fossa level. Diamonds: hyaline cartilage. Red arrows indicate the suprascapular artery.

\section{Evidence}

A recent meta-analysis showed that suprascapular nerve block led to better pain relief than physical therapy or placebo injection alone, but had similar efficacy with glenohumeral joint injection for chronic shoulder pain. ${ }^{37}$ The use of US guidance was consistently more effective than the landmark technique or fluoroscopy guidance. Another meta-analysis revealed that suprascapular nerve block effectively reduced acute postoperative shoulder pain and the incidence of opioidrelated nausea. ${ }^{38}$ However, the beneficial effect was not seen in patients undergoing laparoscopic surgery or thoracotomy.

\section{Axillary nerve Anatomy}

The axillary nerve arises from the posterior cord of the brachial plexus, receiving nerve fibers from the ventral rami of C5 and C6. It passes the junction between the subscapularis and the conjoint (teres major and latissimus dorsi) tendons and wraps around the humeral head to reach the posterior aspect of the shoulder through the quadrilateral space (Figure 6A). The posterior humeral circumflex artery courses beside the axillary artery. The nerve further divides into an anterior branch to innervate the anterior and middle parts 

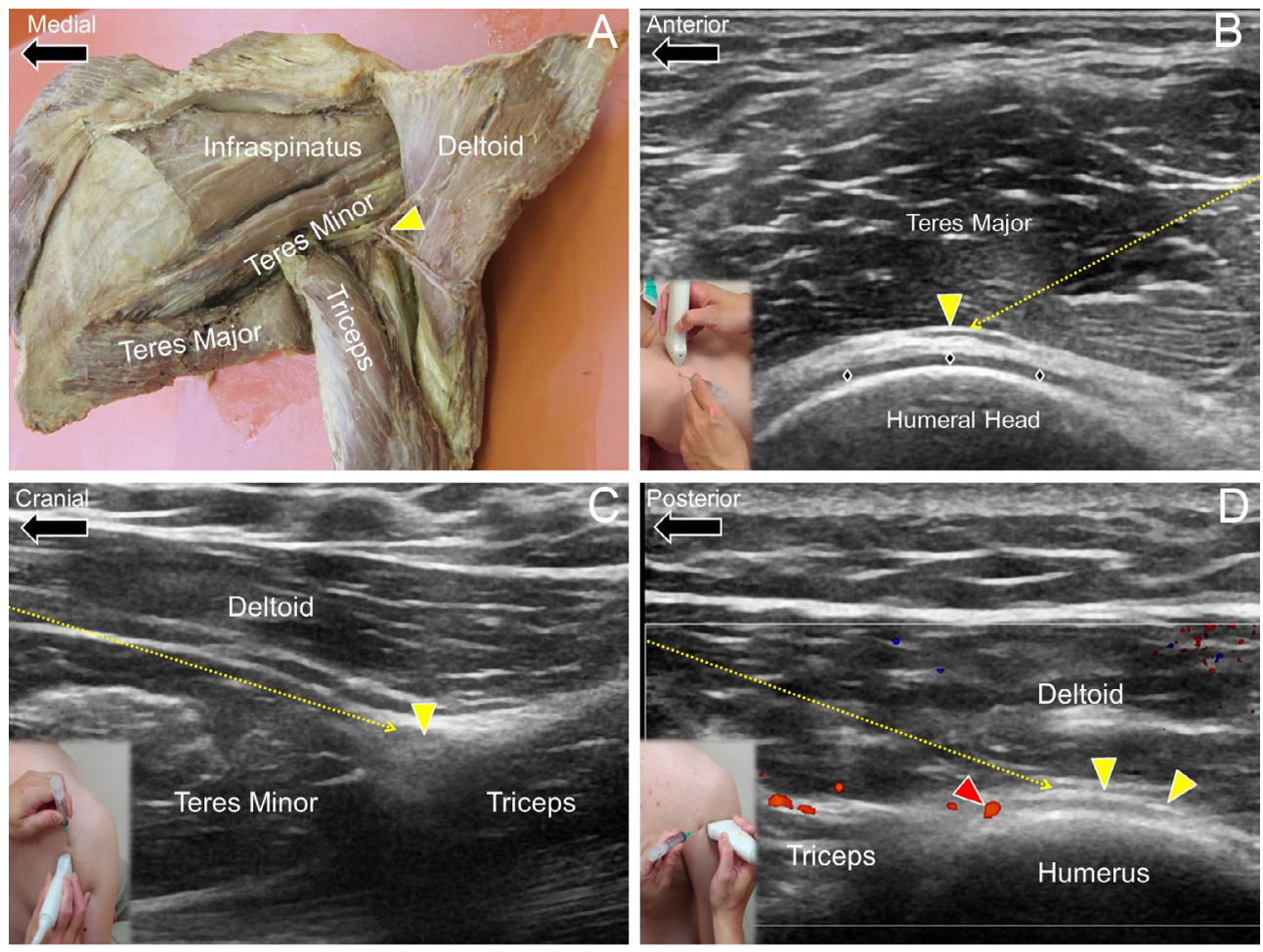

Figure 6 Ultrasound guided injection for the axillary nerve

Notes: (A) Cadaver model of the axillary nerve (yellow arrowhead) at the posterior aspect of the shoulder. (B) US-guided in-plane (dashed arrow) approach to the axillary nerve at the inferior axillary level. Diamonds: hyaline cartilage. (C) US-guided in-plane (dashed arrow) approach to the axillary nerve at the quadrilateral space. (D) US-guided in-plane (dashed arrow) approach to the axillary nerve at the humeral neck level. Red arrowhead shows the posterior lateral humeral circumflex artery. Yellow arrowheads indicate the axillary nerve.

of the deltoid muscle and a posterior branch to supply the posterior part of the deltoid and teres minor muscles. It also provides sensory innervation to the posterior and inferior aspects of the glenohumeral capsule and superior lateral portion of the arm. ${ }^{39}$

\section{US imaging and guided injection technique \\ Infra-axillary level}

The patient lies on the side with full abduction of the shoulder. The transducer is placed on the axillary fold along the axial plane. The axillary nerve is seen wrapped around the humeral head underneath the teres major muscle. The needle is introduced from posterior to anterior using an in-plane approach and a long segment of the proximal part of the nerve can be hydrodissected (Figure 6B, Video S6). ${ }^{40}$

\section{Quadrilateral space level}

The patient is seated with the arm hanging by the side. The transducer is placed along the sagittal plane at the lateral edge of the scapula to visualize the short axis of teres minor muscle. The transducer is then repositioned laterally until the long head of triceps muscle is seen. The axillary nerve is situated in the space bordered by deltoid muscle superficially, teres minor muscle cranially, and the long head of triceps muscle caudally (Figure 6C, Video $\mathrm{S} 6$ ). The needle is introduced from cranial to caudal using an in-plane approach until the needle tip reaches the perineural region. ${ }^{41}$

\section{Superior lateral humerus level}

The transducer is placed along the long axis of the teres minor insertion on the humerus. The axillary nerve appears as a hypoechoic dot caudal to the inferior edge of the teres minor muscle. Once the short axis of the nerve is located, the transducer can be repositioned to align with the long axis of the nerve. The power Doppler mode is used to avoid injury to the posterior humeral circumflex artery. The needle is introduced from posterior to anterior using an in-plane approach and a long segment of the axillary nerve can be hydrodissected (Figure 6D, Video S6).

\section{Evidence}

US-guided axillary nerve block was first introduced by Rothe et al, who targeted the axillary nerve near the quadrilateral space. ${ }^{41}$ Combination of the suprascapular and axillary nerve blocks has been widely applied for the management 
of postoperative shoulder pain and was reported to provide better pain reduction than interscalene brachial plexus block or suprascapular nerve block alone. ${ }^{42,43}$ Due to the proximity of the axillary nerve to the surgical neck of the humerus, the nerve is prone to injury after shoulder trauma and might lead to chronic shoulder pain. A recent anatomic study revealed that the bicipital groove and subacromial-subdeltoid bursa were also innervated by the axillary nerve. ${ }^{44}$ Therefore, in patients with refractory pain, not responsive to injections of the subacromial-subdeltoid bursa or the glenohumeral joint, entrapment of the axillary nerve should be suspected, possibly necessitating a diagnostic block. ${ }^{44}$

\section{Conclusion}

Diagnosis and treatment of shoulder pain is never an easy task, and US imaging enables the physician to thoroughly investigate the underlying pathology and to precisely guide further intervention. Although pathology of the involved tendons, bursas, and joints are common causes of shoulder pain, irritation, or entrapment of nerves that supply the shoulder should also be taken into consideration when pain becomes chronic. In such situations, a combination of peritendinous, intrabursal, intra-articular, and perineural injections may occasionally be required for refractory pain. Finally, it is important to scan the target area routinely by Doppler imaging to prevent possible injury to the adjacent vasculature.

\section{Acknowledgment}

The present study is supported by (1) National Taiwan University Hospital, Bei-Hu Branch, (2) Ministry of Science and Technology (MOST 106-2314-B-002-078-MY2 and 106-2314-B-002-180-MY3), and (3) Taiwan Society of Ultrasound in Medicine.

\section{Disclosure}

The authors report no conflicts of interest in this work.

\section{References}

1. Luime JJ, Koes BW, Hendriksen IJ, et al. Prevalence and incidence of shoulder pain in the general population; a systematic review. Scand J Rheumatol. 2004;33(2):73-81.

2. Hung CY, Chang KV, Özçakar L, Wang TG, Chen WS. Can quantification of biceps peritendinous effusion predict rotator cuff pathologies? A retrospective analysis of 1352 shoulder ultrasound. Am J Phys Med Rehabil. 2016;95(3):161-168.

3. Kostretzis L, Theodoroudis I, Boutsiadis A, Papadakis N, Papadopoulos P. Suprascapular nerve pathology: a review of the literature. Open Orthop J. 2017;11:140-153.

4. Özçakar L, Kara M, Chang KV, et al. Nineteen reasons why physiatrists should do musculoskeletal ultrasound: EURO-MUSCULUS/USPRM recommendations. Am J Phys Med Rehabil. 2015;94(6):e45-e49.
5. Sekizkardes M, Aydin G, Özçakar L. Ultrasound-guided shoulder injection or ultrasound guides the shoulder injection? Standard vs state-of-the-art. Am J Phys Med Rehabil. 2018;97(5):e46.

6. Hsiao MY, Hung CY, Chang KV, Özçakar L. Dynamic ultrasonography of the intra-articular long head biceps tendon and superior labrum. Am J Phys Med Rehabil. 2016;95(11):e183-e184.

7. Chang KV, Wu SH, Lin SH, Shieh JY, Wang TG, Chen WS. Power Doppler presentation of shoulders with biceps disorder. Arch Phys Med Rehabil. 2010;91(4):624-631.

8. Özçakar L, Kara M, Chang KV, et al. EURO-MUSCULUS/USPRM Basic Scanning Protocols for shoulder. Eur J Phys Rehabil Med. 2015;51(4):491-496.

9. Stone TJ, Adler RS. Ultrasound-guided biceps peritendinous injections in the absence of a distended tendon sheath: a novel rotator interval approach. J Ultrasound Med. 2015;34(12):2287-2292.

10. Petscavage-Thomas J, Gustas C. Comparison of ultrasound-guided to fluoroscopy-guided biceps tendon sheath therapeutic injection. J Ultrasound Med. 2016;35(10):2217-2221.

11. Chang KV, Chen WS, Wang TG, Hung CY, Chien KL. Quantitative ultrasound facilitates the exploration of morphological association of the long head biceps tendon with supraspinatus tendon full thickness tear. PLoS One. 2014;9(11):e113803.

12. Chang KV, Chen WS, Wang TG, Hung CY, Chien KL. Associations of sonographic abnormalities of the shoulder with various grades of biceps peritendinous effusion (BPE). Ultrasound Med Biol. 2014;40(2):313-321.

13. Chang KV, Wu WT, Özçakar L. Association of bicipital peritendinous effusion with subacromial impingement: a dynamic ultrasonographic study of 337 shoulders. Sci Rep. 2016;6:38943.

14. Emura K, Arakawa T, Miki A, Terashima T. Anatomical observations of the human acromioclavicular joint. Clin Anat. 2014;27(7): 1046-1052.

15. Salter EG Jr, Nasca RJ, Shelley BS. Anatomical observations on the acromioclavicular joint and supporting ligaments. Am J Sports Med. 1987;15(3):199-206.

16. Saccomanno MF, de Ieso C, Milano G. Acromioclavicular joint instability: anatomy, biomechanics and evaluation. Joints. 2014;2(2): 87-92.

17. Peck E, Lai JK, Pawlina W, Smith J. Accuracy of ultrasound-guided versus palpation-guided acromioclavicular joint injections: a cadaveric study. PM R. 2010;2(9):817-821.

18. Borbas P, Kraus T, Clement H, Grechenig S, Weinberg AM, Heidari N. The influence of ultrasound guidance in the rate of success of acromioclavicular joint injection: an experimental study on human cadavers. J Shoulder Elbow Surg. 2012;21(12):1694-1697.

19. Park KD, Kim TK, Lee J, Lee WY, Ahn JK, Park Y. Palpation versus ultrasound-guided acromioclavicular joint intra-articular corticosteroid injections: a retrospective comparative clinical study. Pain Physician. 2015;18(4):333-341.

20. Sabeti-Aschraf M, Ochsner A, Schueller-Weidekamm C, et al. The infiltration of the AC joint performed by one specialist: ultrasound versus palpation a prospective randomized pilot study. Eur J Radiol. 2010;75(1):e37-e40.

21. Sabeti-Aschraf M, Stotter C, Thaler C, et al. Intra-articular versus periarticular acromioclavicular joint injection: a multicenter, prospective, randomized, controlled trial. Arthroscopy. 2013;29(12):1903-1910.

22. Kennedy MS, Nicholson HD, Woodley SJ. Clinical anatomy of the subacromial and related shoulder bursae: a review of the literature. Clin Anat. 2017;30(2):213-226.

23. Peng PW, Cheng P. Ultrasound-guided interventional procedures in pain medicine: a review of anatomy, sonoanatomy, and procedures. Part III: shoulder. Reg Anesth Pain Med. 2011;36(6):592-605.

24. Chang KV, Wu WT, Han DS, Özçakar L. Static and dynamic shoulder imaging to predict initial effectiveness and recurrence after ultrasoundguided subacromial corticosteroid injections. Arch Phys Med Rehabil. 2017;98(10):1984-1994. 
25. Wu T, Song HX, Dong Y, Li JH. Ultrasound-guided versus blind subacromial-subdeltoid bursa injection in adults with shoulder pain: a systematic review and meta-analysis. Semin Arthritis Rheum. 2015;45(3):374-378.

26. Cole BF, Peters KS, Hackett L, Murrell GA. Ultrasound-guided versus blind subacromial corticosteroid injections for subacromial impingement syndrome: a randomized, double-blind clinical trial. Am J Sports Med. 2016;44(3):702-707.

27. Lee DH, Hong JY, Lee MY, Kwack KS, Yoon SH. Relation between subacromial bursitis on ultrasonography and efficacy of subacromial corticosteroid injection in rotator cuff disease: a prospective comparison study. Arch Phys Med Rehabil. 2017;98(5):881-887.

28. Sugalski MT, Wiater JM, Levine WN, Bigliani LU. An anatomic study of the humeral insertion of the inferior glenohumeral capsule. J Shoulder Elbow Surg. 2005;14(1):91-95.

29. Zhang JY, Fabricant PD, Ishmael CR, Wang JC, Petrigliano FA, Jones KJ. Utilization of platelet-rich plasma for musculoskeletal injuries: an analysis of current treatment trends in the United States. Orthop $J$ Sports Med. 2016;4(12):2325967116676241.

30. Lee SY, Lee KJ, Kim W, Chung SG. Relationships between capsular stiffness and clinical features in adhesive capsulitis of the shoulder. $P M$ R. 2015;7(12):1226-1234.

31. Patel DN, Nayyar S, Hasan S, Khatib O, Sidash S, Jazrawi LM. Comparison of ultrasound-guided versus blind glenohumeral injections: a cadaveric study. J Shoulder Elbow Surg. 2012;21(12):1664-1668.

32. Amber KT, Landy DC, Amber I, Knopf D, Guerra J. Comparing the accuracy of ultrasound versus fluoroscopy in glenohumeral injections: a systematic review and meta-analysis. $J$ Clin Ultrasound. 2014;42(7):411-416.

33. Kim DY, Lee SS, Nomkhondorj O, et al. Comparison between anterior and posterior approaches for ultrasound-guided glenohumeral steroid injection in primary adhesive capsulitis: a randomized controlled trial. $J$ Clin Rheumatol. 2017;23(1):51-57.

34. Wu WT, Chang KV, Han DS, Chang CH, Yang FS, Lin CP. Effectiveness of glenohumeral joint dilatation for treatment of frozen shoulder: a systematic review and meta-analysis of randomized controlled trials. Sci Rep. 2017;7(1):10507.
35. Laumonerie $\mathrm{P}$, Lapègue F, Chantalat E, Sans N, Mansat P, Faruch M. Description and ultrasound targeting of the origin of the suprascapular nerve. Clin Anat. 2017;30(6):747-752.

36. Chang KV, Hung CY, Wang TG, Yang RS, Sun WZ, Lin CP. Ultrasoundguided proximal suprascapular nerve block with radiofrequency lesioning for patients with malignancy-associated recalcitrant shoulder pain. J Ultrasound Med. 2015;34(11):2099-2105.

37. Chang KV, Hung CY, Wu WT, Han DS, Yang RS, Lin CP. Comparison of the effectiveness of suprascapular nerve block with physical therapy, placebo, and intra-articular injection in management of chronic shoulder pain: a meta-analysis of randomized controlled trials. Arch Phys Med Rehabil. 2016;97(8):1366-1380.

38. Chang KV, Wu WT, Hung CY, et al. Comparative effectiveness of suprascapular nerve block in the relief of acute post-operative shoulder pain: a systematic review and meta-analysis. Pain Physician. 2016;19(7):445-456.

39. Leechavengvongs S, Teerawutthichaikit T, Witoonchart K, et al. Surgical anatomy of the axillary nerve branches to the deltoid muscle. Clin Anat. 2015;28(1):118-122.

40. Chang KV, Lin CP, Lin CS, Wu WT, Özçakar L. A novel approach for ultrasound guided axillary nerve block: the inferior axilla technique. Med Ultrason. 2017;19(4):457-461.

41. Rothe C, Asghar S, Andersen HL, Christensen JK, Lange KH. Ultrasound-guided block of the axillary nerve: a volunteer study of a new method. Acta Anaesthesiol Scand. 2011;55(5):565-570.

42. Lee JJ, Kim DY, Hwang JT, et al. Effect of ultrasonographically guided axillary nerve block combined with suprascapular nerve block in arthroscopic rotator cuff repair: a randomized controlled trial. Arthroscopy. 2014;30(8):906-914.

43. Dhir S, Sondekoppam RV, Sharma R, Ganapathy S, Athwal GS. A comparison of combined suprascapular and axillary nerve blocks to interscalene nerve block for analgesia in arthroscopic shoulder surgery: an equivalence study. Reg Anesth Pain Med. 2016;41(5):564-571.

44. Nasu H, Nimura A, Yamaguchi K, Akita K. Distribution of the axillary nerve to the subacromial bursa and the area around the long head of the biceps tendon. Knee Surg Sports Traumatol Arthrosc. 2015;23(9):2651-2657.
Journal of Pain Research

\section{Publish your work in this journal}

The Journal of Pain Research is an international, peer reviewed, open access, online journal that welcomes laboratory and clinical findings in the fields of pain research and the prevention and management of pain. Original research, reviews, symposium reports, hypothesis formation and commentaries are all considered for publication.

\section{Dovepress}

The manuscript management system is completely online and includes a very quick and fair peer-review system, which is all easy to use. Visit http://www.dovepress.com/testimonials.php to read real quotes from published authors. 